\title{
On the dynamics of stock price bubbles: comments on a model by Miao and Wang
}

\author{
Gerhard Sorger $^{1}[$ \\ Published online: 18 September 2019 \\ (c) The Author(s) 2019
}

\begin{abstract}
We consider the model by Miao and Wang (Am Econ Rev 108:2590-2628, 2018), in which endogenous collateral constraints may generate stock price bubbles. Whereas Miao and Wang (2018) characterize the local dynamics around stationary equilibria only under the assumption of risk neutral households, we extend this characterization to the case of risk aversion.
\end{abstract}

Keywords Stock price bubbles · Risk aversion - Local stability analysis

\section{Introduction}

Miao and Wang (2018) develop a theory of rational stock price bubbles that rests on endogenous credit constraints. The main results of the paper are derived under the assumption that the households are risk neutral, whereas for the case of risk averse households only the characterization of stationary equilibria is conducted. ${ }^{1}$ The purpose of the present note is to fill this gap, i.e., to provide a complete analysis of the local dynamics in a version of the model with risk averse households.

This paper was presented at the symposium on the occasion of the award of the "Wissenschaftspreis der Österreichischen Forschungsgemeinschaft" to Gustav Feichtinger. The paper is dedicated to Gustav, whom I thank for his support and guidance during the early stages of my career. I acknowledge valuable comments by two anonymous referees.

Gerhard Sorger

gerhard.sorger@univie.ac.at

1 Department of Economics, University of Vienna, Oskar Morgenstern-Platz 1, 1090 Vienna, Austria

1 Regarding the model with risk averse households, Miao and Wang (2018) write in Appendix D: "We are unable to derive analytical results for local dynamics because the equilibrium system contains five equations, but it is straightforward to derive numerical solutions." 
The model by Miao and Wang (2018) is a notable contribution for various reasons. First of all, it shows that endogenous credit constraints can generate bubbles that are consistent with the transversality condition at infinity. More specifically, because the presence of a bubble relaxes the credit constraints for firms, the bubble component of the asset price does not have to grow at the rate of interest. Second, the model by Miao and Wang (2018) directly addresses bubbles on markets for common stock, whereas most of the previous literature has dealt with bubbles on intrinsically useless assets. The fact that the assets under consideration are productive ones opens the door for an important and intuitively plausible feedback mechanism. If the stock price of a company contains a positive bubble component, the firm's assets have a higher value than in a bubbleless equilibrium. Since the firm can use these assets as collateral, it is able to obtain more credit which, in turn, allows the firm to invest more. Thus, and this is the third notable feature of the model, the bubbly equilibrium features higher capital and more output than the bubbleless one, i.e., there is a crowding-in effect. Miao and Wang $(2012,2014)$ have already extended their theory to economies with idiosyncratic productivity shocks, multiple production sectors, and endogenous growth.

The rest of this paper is organized as follows. In Sect. 2 we present the basic model with risk averse households. Section 3 analyzes competitive equilibria: stationary equilibria are characterized in Sect. 3.1, whereas the local dynamics around these stationary equilibria are studied in Sect. 3.2. To overcome the difficulties mentioned by Miao and Wang (2018) (see Footnote 1 above), we use the output market clearing condition to express the real interest rate as a function of the aggregate capital stock and the two components of the stock price (Tobin's marginal $Q$ and the bubble component). This eliminates aggregate consumption from all equilibrium conditions except for the Euler equation. Consequently, we are left with a three-dimensional system of ordinary differential equations which is analytically tractable. The analysis demonstrates that the results about the local dynamics that have been derived by Miao and Wang (2018) for the economy with risk neutral households carry over to the case of risk averse households. Finally, Sect. 4 concludes the paper.

\section{Model formulation}

Time is modeled as a continuous variable on the time domain $\mathbb{R}_{+}$. We consider an economy that is populated by households and firms. Firms use the input factors capital and labor to produce a single output good. The latter can be used for consumption and for investment and it serves as numeraire good. Households are endowed with labor and they own the firms. Firms own their capital and rent labor services from households. There a two assets in the economy: bonds, which are available in zero net supply, and firm equity.

\subsection{Households}

There exists a unit interval of identical and infinitely-lived households. The representative household is endowed with one unit of labor per period and it owns equal shares 
of all firms. Furthermore, it has the time-preference rate $\rho>0$ and the instantaneous utility function

$$
u(c)=\left\{\begin{array}{cc}
\frac{c^{1-\theta}-1}{1-\theta} & \text { if } \theta \neq 1 \\
\ln c & \text { if } \theta=1
\end{array}\right.
$$

where $\theta>0$ is the inverse of the elasticity of intertemporal substitution. The household maximizes its lifetime utility

$$
\int_{0}^{+\infty} e^{-\rho t} u(c(t)) \mathrm{d} t
$$

subject to the budget constraint

$$
\dot{b}(t)+p(t) \dot{s}(t)+c(t)=r(t) b(t)+d(t) s(t)+w(t)
$$

and the initial conditions

$$
s(0)=1 \text { and } b(0)=0 .
$$

The choice variables of the household are its consumption rate $c(t)$, share holdings $s(t)$, and bond holdings $b(t)$. On the other hand, the household takes the variables $p(t)$, $r(t), d(t)$, and $w(t)$ as given. These variables denote the time- $t$ share price, interest rate, dividend payment per share, and the wage rate, respectively.

Absence of arbitrage requires that

$$
r(t)=\frac{d(t)+\dot{p}(t)}{p(t)}
$$

holds for all $t$. We denote total financial wealth of the representative household at time $t$ by $a(t)$, that is,

$$
a(t)=b(t)+p(t) s(t)
$$

Using this notation, we can rewrite the flow budget constraint from above as

$$
\dot{a}(t)=r(t) a(t)+w(t)-c(t) .
$$

It is well known that the behavior of the representative household is completely described by constraint (1), the initial value $a(0)$, the Euler equation

$$
\frac{\dot{c}(t)}{c(t)}=\frac{r(t)-\rho}{\theta},
$$


and the transversality condition

$$
\lim _{t \rightarrow+\infty} e^{-\int_{0}^{t} r(s) \mathrm{d} s} a(t)=0
$$

Note that $a(0)=p(0)$ is the stock market value of all firms at time 0 . Thus, even if the representative household takes this initial value as given, $a(0)$ is not an exogenous parameter of the model but an endogenously determined variable.

\subsection{Firms}

There exists a unit interval of infinitely-lived firms, which produce output from capital and labor. The firms own their capital and they rent the labor services from the households. Consider any individual firm $j \in[0,1]$. Output of firm $j$ is given by

$$
y_{j}(t)=k_{j}(t)^{\alpha} \ell_{j}(t)^{1-\alpha}
$$

where $k_{j}(t)$ and $\ell_{j}(t)$ denote the time- $t$ input quantities of capital and labor, respectively. The firm can choose its employment $\ell_{j}(t) \geq 0$ at any point in time. Investment, however, is assumed to be lumpy. Formally, we assume that firm $j$ can invest only at discrete instants of time $t_{j, 1}, t_{j, 2}, \ldots$, where $\left(t_{j, n}\right)_{n=1}^{+\infty}$ is a Poisson process with arrival rate $\lambda$. We choose the unit of time in such a way that $\lambda=1 .^{2}$ The investment opportunities are independent across firms. Denoting by $\delta$ the rate of capital depreciation and by $i_{j}(t)$ the (lumpy) investment at time $t$, it follows that firm $j$ 's capital stock evolves according to

$$
\dot{k}_{j}(t)=-\delta k_{j}(t)
$$

for $t \notin\left\{t_{j, n} \mid n=1,2, \ldots\right\}$ and

$$
\lim _{s \searrow t} k_{j}(s)=k_{j}(t)+i_{j}(t)
$$

for $t \in\left\{t_{j, n} \mid n=1,2, \ldots\right\}$. Thus, we assume that the function $k_{j}$ is piecewise continuously differentiable with jump discontinuities at the investment opportunities and that it is continuous from the left everywhere. Finally, we assume that all firms are initially endowed with $\bar{k}>0$ units of capital.

Firms maximize their shareholder value. The maximal stock market value of firm $j$ when it owns $k$ units of capital at time $s$ is

$$
\begin{aligned}
V(k, s)= & \max \mathbb{E}_{S}\left[\int_{s}^{+\infty} e^{-\int_{s}^{t} r(\tau) \mathrm{d} \tau}\left[y_{j}(t)-w(t) \ell_{j}(t)\right] \mathrm{d} t\right. \\
& \left.-\sum_{\left\{n \mid t_{j, n} \geq s\right\}} e^{-\int_{s}^{t_{j}, n} r(\tau) \mathrm{d} \tau} i_{j}\left(t_{j, n}\right)\right]
\end{aligned}
$$

\footnotetext{
2 This normalization implies that the time-preference rate $\rho$ and the depreciation rate $\delta$ have to be interpreted relative to the Poisson arrival rate. The results for the model without the normalization $\lambda=1$ can therefore be obtained by replacing $\rho$ and $\delta$ by $\rho / \lambda$ and $\delta / \lambda$, respectively.
} 
where the maximum is taken over employment and investment subject to constraints (4)-(6), the initial condition $k(s)=k$, and the control constraints $\ell_{j}(t) \geq 0$ and

$$
-k_{j}(t) \leq i_{j}(t) \leq V\left(\xi k_{j}(t), t\right)
$$

for all $t$.

According to its definition, $V(k, s)$ is the expected and discounted value of the firm's future profits. The constraint $\ell_{j}(t) \geq 0$ restricts employment to be non-negative and the constraint $i_{j}(t) \geq-k_{j}(t)$ says that disinvestment is possible but that the capital stock of the firm must remain non-negative. The crucial constraint is $i_{j}(t) \leq$ $V\left(\xi k_{j}(t), t\right)$, which is interpreted as a collateral constraint. The parameter $\xi$ satisfies $0<\xi \leq 1$. Firms cannot raise additional equity and are therefore forced to finance their investments via loans. We do not model the credit market explicitly but simply assume that financial frictions or asymmetric information imply that the investment of firm $j$ must not exceed the stock market value of a hypothetical firm owning the fraction $\xi$ of firm $j$ 's current capital stock. ${ }^{3}$

The Hamilton-Jacobi-Bellman equation (HJB equation) for the above optimization problem is ${ }^{4}$

$$
\begin{aligned}
& r(t) V\left(k_{j}, t\right)-V_{2}\left(k_{j}, t\right) \\
& \quad=\max \left\{k_{j}^{\alpha} \ell_{j}^{1-\alpha}-w(t) \ell_{j}-V_{1}\left(k_{j}, t\right) \delta k_{j}+\left[V\left(k_{j}+i_{j}, t\right)-V\left(k_{j}, t\right)-i_{j}\right]\right\} .
\end{aligned}
$$

Carrying out the maximization with respect to labor input in the HJB equation yields

$$
\ell_{j}=\left[\frac{1-\alpha}{w(t)}\right]^{1 / \alpha} k_{j}
$$

Substituting this back into the HJB equation, the latter simplifies to

$$
\begin{aligned}
& r(t) V\left(k_{j}, t\right)-V_{2}\left(k_{j}, t\right) \\
& \quad=\max \left\{\alpha\left[\frac{1-\alpha}{w(t)}\right]^{(1-\alpha) / \alpha} k_{j}-V_{1}\left(k_{j}, t\right) \delta k_{j}+V\left(k_{j}+i_{j}, t\right)-V\left(k_{j}, t\right)-i_{j}\right\} .
\end{aligned}
$$

Given the structure of this equation, we conjecture a linear affine value function of the form $V\left(k_{j}, t\right)=Q(t) k_{j}+q(t)$. The variable $Q(t)$ denotes the value of capital inside the firm at time $t$, i.e., $Q(t)$ is Tobin's marginal $Q$. The variable $q(t)$ describes those components of the firm's value that do not originate from its capital stock. For this reason, an equilibrium with $q(t) \neq 0$ is said to contain a bubble. If $q(t)=0$, then it follows that $Q(t)$ coincides with Tobin's average $Q$.

\footnotetext{
${ }^{3}$ For further explanations of the constraint $i_{j}(t) \leq V\left(\xi k_{j}(t), t\right)$ we refer to Miao and Wang (2018). They interpret $(1-\xi) k_{j}(t)$ as an efficiency loss that occurs when the lender takes over the firm or, alternatively, as a default cost including, for example, legal expenses.

${ }^{4}$ Recall that we have normalized the arrival rate of investment opportunities, $\lambda$, by 1 . The bracketed term $V\left(k_{j}+i_{j}, t\right)-V\left(k_{j}, t\right)-i_{j}$ in the HJB equation is multiplied by this arrival rate. The maximization in the HJB equation is over $\ell_{j} \geq 0$ and over $i_{j} \in\left[-k_{j}, V\left(\xi k_{j}, t\right)\right]$.
} 
With the linear specification of the optimal value function introduced above, the HJB equation turns into

$$
\begin{aligned}
r(t) & {\left[Q(t) k_{j}+q(t)\right]-\dot{Q}(t) k_{j}-\dot{q}(t) } \\
= & \max \left\{\alpha\left[\frac{1-\alpha}{w(t)}\right]^{(1-\alpha) / \alpha} k_{j}-Q(t) \delta k_{j}\right. \\
& \left.+[Q(t)-1] i_{j} \mid-k_{j} \leq i_{j} \leq \xi Q(t) k_{j}+q(t)\right\} \\
= & \begin{cases}\alpha\left[\frac{1-\alpha}{w(t)}\right]^{(1-\alpha) / \alpha} k_{j}+[1-(1+\delta) Q(t)] k_{j} & \text { if } Q(t)<1, \\
\alpha\left[\frac{1-\alpha}{w(t)}\right]^{(1-\alpha) / \alpha} k_{j}-\delta Q(t) k_{j}+[Q(t)-1]\left[\xi Q(t) k_{j}+q(t)\right] & \text { if } Q(t) \geq 1 .\end{cases}
\end{aligned}
$$

The optimal investment rate is given by

$$
i_{j}= \begin{cases}-k_{j} & \text { if } Q(t)<1 \\ \xi Q(t) k_{j}+q(t) & \text { if } Q(t)>1\end{cases}
$$

In the case where $Q(t)=1$ holds, the investment rate is not uniquely pinned down by the above maximization problem and any value $i_{j} \in\left[-k_{j}, \xi Q(t) k_{j}+q(t)\right]$ is possible. Since the HJB equation has to hold for all $t$ and all $k_{j}$ it follows that

$$
\begin{aligned}
& \dot{Q}(t)= \begin{cases}{[1+\delta+r(t)] Q(t)-1-\alpha\left[\frac{1-\alpha}{w(t)}\right]^{(1-\alpha) / \alpha}} & \text { if } Q(t)<1, \\
{[r(t)+\delta] Q(t)-\xi Q(t)[Q(t)-1]-\alpha\left[\frac{1-\alpha}{w(t)}\right]^{(1-\alpha) / \alpha}} & \text { if } Q(t) \geq 1,\end{cases} \\
& \dot{q}(t)= \begin{cases}r(t) q(t) & \text { if } Q(t)<1, \\
r(t) q(t)-[Q(t)-1] q(t) & \text { if } Q(t) \geq 1\end{cases}
\end{aligned}
$$

The behavior of the firms is completely described by Eqs. (4)-(10) along with the initial condition $k(0)=\bar{k}$.

\subsection{Aggregation and market clearing}

Defining the aggregate variables $y(t)=\int_{0}^{1} y_{j}(t) \mathrm{d} j, k(t)=\int_{0}^{1} k_{j}(t) \mathrm{d} j, \ell(t)=$ $\int_{0}^{1} \ell_{j}(t) \mathrm{d} j$, and $i(t)=\int_{0}^{1} i_{j}(t) \mathrm{d} j$ and recalling that the arrival rate for investment opportunities has been normalized to 1 , it follows from (4)-(8) that

$$
\begin{aligned}
& y(t)=\left[\frac{1-\alpha}{w(t)}\right]^{(1-\alpha) / \alpha} k(t)=k(t)^{\alpha} \ell(t)^{1-\alpha}, \\
& \dot{k}(t)=i(t)-\delta k(t),
\end{aligned}
$$




$$
\begin{aligned}
\ell(t) & =\left[\frac{1-\alpha}{w(t)}\right]^{1 / \alpha} k(t), \\
i(t) & = \begin{cases}-k(t) & \text { if } Q(t)<1, \\
\xi Q(t) k(t)+q(t) & \text { if } Q(t)>1 .\end{cases}
\end{aligned}
$$

The markets for labor services, output, and assets clear if

$$
\begin{aligned}
& \ell(t)=1, \\
& y(t)=c(t)+i(t), \\
& a(t)=V(k(t), t)+b(t)=Q(t) k(t)+q(t) .
\end{aligned}
$$

In the last of these equations we have used the assumption that bonds are available in zero net supply.

Finally, we have to make sure that households keep their firms running. This will be the case if the market value of the firms held by the representative household is at least as large as the market value of the capital installed in those firms. Since the value of capital outside the firms is equal to 1 and since the representative household owns equal shares of all firms, this means that $V(k(t), t)=Q(t) k(t)+q(t) \geq k(t)$ has to hold. A sufficient condition for this inequality is that

$$
Q(t) \geq 1 \text { and } q(t) \geq 0
$$

are satisfied for all $t .^{5}$

\section{Equilibrium analysis}

A family of functions $(c, a, k, \ell, y, i, r, w, Q, q)$ is called an equilibrium from $\bar{k}$ if it satisfies conditions (1)-(3) and (9)-(18) for all $t$ and if $k(0)=\bar{k}$ holds. A family of constant functions ( $c, a, k, \ell, y, i, r, w, Q, q)$ is called a stationary equilibrium if it satisfies (1)-(3) and (9)-(18) for all $t$. An equilibrium or a stationary equilibrium is called non-degenerate if $y(t)>0, k(t)>0$, and $c(t)>0$ hold for all $t$.

According to Walras' law, one of the market clearing conditions or budget constraints is redundant. Therefore, we shall disregard the flow budget equation (1) in the following analysis. Using (17) it follows that (3) holds if

$$
\lim _{t \rightarrow+\infty} e^{-\int_{0}^{t} r(s) \mathrm{d} s} Q(t) k(t)=\lim _{t \rightarrow+\infty} e^{-\int_{0}^{t} r(s) \mathrm{d} s} q(t)=0 .
$$

Finally, we note that (11), (13), and (15) together imply that

$$
w(t)=(1-\alpha) k(t)^{\alpha},
$$

\footnotetext{
5 The inequalities in (18) are of course not necessary for $V(k(t), t) \geq k(t)$ to hold. Neither do we know whether the linear specification of the value function $V$ is the only one that is consistent with equilibrium. We follow Miao and Wang $(2012,2014,2018)$ by considering only those equilibria with linear value functions satisfying (18).
} 


$$
y(t)=k(t)^{\alpha}
$$

hold. Consequently, we can combine (9) and (18) to obtain

$$
\dot{Q}(t)=[r(t)+\delta] Q(t)-\xi Q(t)[Q(t)-1]-\alpha k(t)^{\alpha-1} .
$$

\subsection{Non-degenerate stationary equilibria}

In the present subsection we characterize and compare all non-degenerate stationary equilibria of the economy under consideration.

Theorem 1 (a) A non-degenerate stationary equilibrium satisfying $Q(t)=1$ exist if and only if $\xi \geq \delta$ holds. This stationary equilibrium is given by ${ }^{6}$

$$
\begin{aligned}
k(t) & =k_{*}=\left(\frac{\alpha}{\rho+\delta}\right)^{1 /(1-\alpha)} \\
c(t) & =c_{*}=\left(1-\frac{\alpha \delta}{\rho+\delta}\right)\left(\frac{\alpha}{\rho+\delta}\right)^{\alpha /(1-\alpha)}, \\
Q(t) & =Q_{*}=1 \\
q(t) & =q_{*}=0 \\
r(t) & =r_{*}=\rho .
\end{aligned}
$$

(b) A non-degenerate stationary equilibrium satisfying $Q(t)>1$ and $q(t)=0$ exist if and only if $\xi<\delta$ holds. This stationary equilibrium is given by

$$
\begin{aligned}
k(t) & =k_{0}=\left[\frac{\alpha \xi}{\delta(\rho+\xi)}\right]^{1 /(1-\alpha)} \\
c(t) & =c_{0}=\left(1-\frac{\alpha \xi}{\rho+\xi}\right)\left[\frac{\alpha \xi}{\delta(\rho+\xi)}\right]^{\alpha /(1-\alpha)}, \\
Q(t) & =Q_{0}=\frac{\delta}{\xi} \\
q(t) & =q_{0}=0 \\
r(t) & =r_{0}=\rho
\end{aligned}
$$

(c) A non-degenerate stationary equilibrium satisfying $Q(t)>1$ and $q(t)>0$ exist if and only if $\xi<\delta /(1+\rho)$ holds. This stationary equilibrium is given by ${ }^{7}$

$$
k(t)=k_{+}=\left[\frac{\alpha}{(1+\rho)[(1-\xi) \rho+\delta]}\right]^{1 /(1-\alpha)},
$$

\footnotetext{
${ }^{6}$ Here and in the rest of the theorem we only state the equilibrium values for $k(t), c(t), Q(t), q(t)$, and $r(t)$. The equilibrium values of the remaining variables can easily be computed from the stated values and the equilibrium conditions.

7 The subscript + in the following formulas indicates that in this stationary equilibrium $q(t)$ is positive, whereas the subscript ${ }_{0}$ in part (b) of the theorem meant that $q(t)=0$.
} 


$$
\begin{aligned}
& c(t)=c_{+}=\left[1-\frac{\alpha \delta}{(1+\rho)[(1-\xi) \rho+\delta]}\right]\left[\frac{\alpha}{(1+\rho)[(1-\xi) \rho+\delta]}\right]^{\alpha /(1-\alpha)}, \\
& Q(t)=Q_{+}=1+\rho, \\
& q(t)=q_{+}=[\delta-\xi(1+\rho)]\left[\frac{\alpha}{(1+\rho)[(1-\xi) \rho+\delta]}\right]^{1 /(1-\alpha)}, \\
& r(t)=r_{+}=\rho .
\end{aligned}
$$

Proof First we observe that the Euler equation (2) implies that in every non-degenerate stationary equilibrium the interest rate is given by $r(t)=\rho$.

(a) Suppose now that $Q(t)=1$ holds for all $t$. In this case (10) shows that $q(t)=0$ must hold for all $t$ and (22) implies that $k(t)=[\alpha /(\delta+\rho)]^{1 /(1-\alpha)}$. From (12) we obtain $i(t)=\delta k(t) \geq 0$ so that (16) and (21) lead to

$$
c(t)=k(t)^{\alpha}-\delta k(t)=\left(1-\frac{\alpha \delta}{\delta+\rho}\right)\left(\frac{\alpha}{\delta+\rho}\right)^{\alpha /(1-\alpha)} .
$$

The collateral constraint says that $i(t)=\delta k(t) \leq V(\xi k(t))=\xi k(t)$ and it follows that $\delta \leq \xi$ must hold. Finally, we observe that the transversality conditions in (19) hold trivially because of the constancy of $k(t), Q(t)$, and $q(t)$ and because of $r(t)=\rho>0$.

(b) Suppose that $Q(t)>1$ and $q(t)=0$ hold. From Eqs. (12) and (14) it follows that $k(t)=0$ or $Q(t)=\delta / \xi$ must hold. The former case is not consistent with the non-degeneracy of the stationary equilibrium and is therefore discarded. The latter case requires the parameter restriction $\xi<\delta$. Substituting $r(t)=\rho$ and $Q(t)=\delta / \xi$ into Eq. (22), we obtain

$$
k(t)=\left[\frac{\alpha \xi}{\delta(\rho+\xi)}\right]^{1 /(1-\alpha)} .
$$

Now we can use conditions (12), (16), and (21) to compute

$$
c(t)=k_{0}^{\alpha}-\delta k_{0}=\left[1-\frac{\alpha \xi}{\rho+\xi}\right]\left[\frac{\alpha \xi}{\delta(\rho+\xi)}\right]^{\alpha /(1-\alpha)}
$$

Non-negativity of investment and the validity of the transversality conditions in (19) can be shown in the same way as in part (a).

(c) Finally, assume that $Q(t)>1$ and $q(t)>0$ are satisfied. It follows from (10) and $r(t)=\rho$ that $Q(t)=1+\rho$ holds. Substituting this value along with $r(t)=\rho$ into (22) we obtain

$$
k(t)=\left[\frac{\alpha}{(1+\rho)[\delta+\rho(1-\xi)]}\right]^{1 /(1-\alpha)} .
$$


Note that the restriction $\xi<\delta /(1+\rho)$ stated in the theorem implies trivially that $\xi<(1+\delta+\rho) /(1+\rho)$ holds which, in turn, is equivalent to

$$
(1+\rho)[\delta+\rho(1-\xi)]>\delta .
$$

This shows that the above value for $k(t)$ is well defined and positive. Substituting the values for $Q(t)$ and $k(t)$ into (12) and (14) we obtain

$$
q(t)=[\delta-\xi(1+\rho)]\left[\frac{\alpha}{(1+\rho)[\delta+\rho(1-\xi)]}\right]^{1 /(1-\alpha)} .
$$

The constraint $q(t)>0$ implies that $\xi<\delta /(1+\rho)$ is satisfied. By substituting the above values into Eq. (16) we obtain

$$
c(t)=\left[1-\frac{\alpha \delta}{(1+\rho)[\delta+\rho(1-\xi)]}\right]\left[\frac{\alpha}{(1+\rho)[\delta+\rho(1-\xi)]}\right]^{\alpha /(1-\alpha)} .
$$

Using (23) it is straightforward to see that $c(t)$ is strictly positive. The nonnegativity constraint on investment as well as the transversality conditions in (19) hold for the same reasons that have already been mentioned above.

The following lemma describes how the stationary equilibria listed in Theorem 1 depend on the parameter $\xi$. We also demonstrate that the capital stock in all nondegenerate stationary equilibria is lower than the Golden Rule capital stock, which is given by $k^{\mathrm{GR}}=(\alpha / \delta)^{1 /(1-\alpha)}$.

Lemma 1 (a) It holds that

$$
\max \left\{k_{*}, k_{0}, k_{+}\right\}<k^{\mathrm{GR}} .
$$

(b) It holds that $k_{0}$ and $c_{0}$ are strictly increasing with respect to $\xi \in(0, \delta)$.

(c) It holds that $k_{+}$and $c_{+}$are strictly increasing with respect to $\xi \in(0, \delta /(1+\rho))$.

(d) If $\xi=1$, then it follows that $k_{0}=k_{+}$and $c_{0}=c_{+}$.

(e) If $\xi<1$, then it holds that $k_{0}<k_{+}$and $c_{0}<c_{+}$.

Proof (a) The inequalities $k_{*}<k^{\mathrm{GR}}, k_{0}<k^{\mathrm{GR}}$, and $k_{+}<k^{\mathrm{GR}}$ follow immediately from Theorem 1 and the parameter restrictions $\rho>0, \xi \in(0,1)$, and $\alpha \in(0,1)$.

(b) It is obvious from Theorem 1(b) that $k_{0}$ is strictly increasing with respect to $\xi$. Defining the function $g: \mathbb{R}_{+} \mapsto \mathbb{R}$ by $g(k)=k^{\alpha}-\delta k$ it holds that $c_{0}=g\left(k_{0}\right)$. Moreover, we have $g^{\prime}(k)=\alpha k^{\alpha-1}-\delta>0$ for all $k \in\left(0, k^{\mathrm{GR}}\right)$ and it follows therefore that $g$ is strictly increasing on the interval $\left(0, k^{\mathrm{GR}}\right)$. Combining these observations and the fact that the function $g$ is independent of $\xi$, one can see that $c_{0}$ is strictly increasing with respect to $\xi$.

(c) Part (c) of the lemma is obvious from Theorem 1(c) and $c_{+}=g\left(k_{+}\right)$.

(d) This part is obvious from Theorem 1(b, c). 
(e) Let us define the parameter $\bar{\xi}=1+\delta / \rho$ and note that $\max \{1, \delta /(1+\rho)\}<\bar{\xi}$ holds. Furthermore, we define the functions $\kappa_{0}:[0, \bar{\xi}) \mapsto \mathbb{R}$ and $\kappa_{+}:[0, \bar{\xi}) \mapsto \mathbb{R}$ by

$$
\kappa_{0}(\xi)=\frac{\xi}{\delta(\rho+\xi)}
$$

and

$$
\kappa_{+}(\xi)=\frac{1}{(1+\rho)[(1-\xi) \rho+\delta]},
$$

respectively. Both of these functions are continuously differentiable and strictly increasing, $\kappa_{0}$ is strictly concave, and $\kappa_{+}$is strictly convex. Finally, we have $\kappa_{0}(0)=0<\kappa_{+}(0), \kappa_{0}(1)=\kappa_{+}(1)$, and $\kappa_{0}(\delta /(1+\rho))=\kappa_{+}(\delta /(1+\rho))$. These properties imply that the graphs of $\kappa_{0}$ and $\kappa_{+}$intersect only in the points $\xi=1$ and $\xi=\delta /(1+\rho)$ and that $\kappa_{0}(\xi)<\kappa_{+}(\xi)$ holds for all $\xi \in(0, \min \{1, \delta /(1+\rho)\})$. The proof of part (e) is completed by noting that $k_{0}=\left[\alpha \kappa_{0}(\xi)\right]^{1 /(1-\alpha)}, k_{+}=$ $\left[\alpha \kappa_{+}(\xi)\right]^{1 /(1-\alpha)}, c_{0}=g\left(k_{0}\right)$, and $c_{+}=g\left(k_{+}\right)$.

Figure 1 illustrates the results stated in Theorem 1 and Lemma 1 for the parameter values $\alpha=1 / 3, \delta=2 / 3$, and $\rho=1$. Note that the parameter values are such that $\delta /(1+\rho)=1 / 3<1$ holds, such that the bubbly stationary equilibrium does not exist for $\xi=1$. Indeed, there exist two non-degenerate stationary equilibria (one of which is bubbly) whenever $\xi$ is smaller than $\delta /(1+\rho)=1 / 3$.

In Fig. 2 we show the situation when $\delta /(1+\rho)>1$. More specifically, the parameter values are $\alpha=1 / 3, \delta=3$, and $\rho=1$. Since $\xi \leq 1$ and $\delta>1+\rho>1$ is satisfied, it

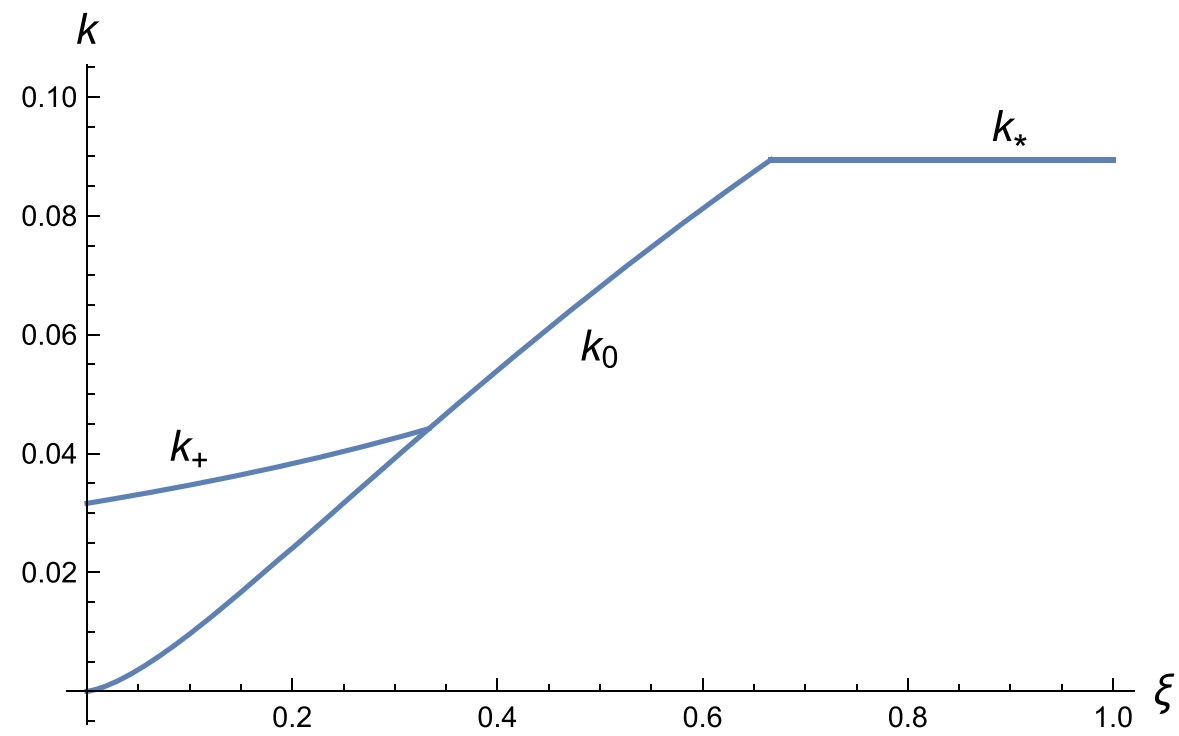

Fig. 1 Capital stocks in the stationary states for $\alpha=1 / 3, \delta=2 / 3$, and $\rho=1$ 


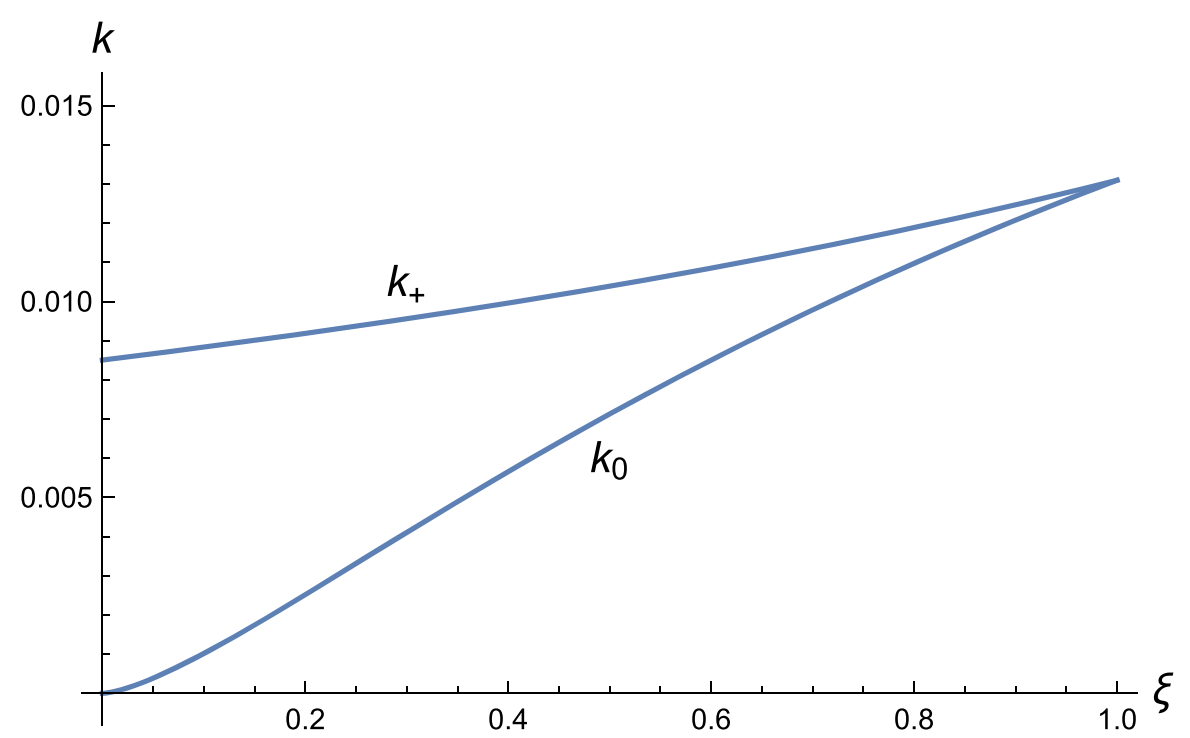

Fig. 2 Capital stocks in the stationary states for $\alpha=1 / 3, \delta=3$, and $\rho=1$

follows from Theorem 1(a) that there exists no non-degenerate stationary equilibrium with $Q(t)=1$. In every non-degenerate stationary equilibrium the collateral constraint is binding.

An interesting special case is $\xi=1$, because we have $k_{0}=k_{+}$and $c_{0}=c_{+}$despite the fact that $Q_{0}=\delta>1+\rho=Q_{+}$and $q_{0}=0<(\delta-1-\rho) k_{+}=q_{+}$. In this case it holds furthermore that $Q_{0} k_{0}+q_{0}=Q_{+} k_{+}+q_{+}$. In other words, the two stationary equilibrium allocations as well as their supporting price systems coincide, but the stock market price of firms can be interpreted to be bubbleless $\left(q_{0}=0\right)$ or to have a positive bubble component $q_{+}>0$.

\subsection{Local dynamics}

In this subsection we study the local dynamics around the stationary equilibria $\left(k_{0}, c_{0}, Q_{0}, q_{0}, r_{0}\right)$ and $\left(k_{+}, c_{+}, Q_{+}, q_{+}, r_{+}\right)$. We focus on these two stationary equilibria because they are the ones for which the collateral constraint is binding.

In the first step we reduce the equilibrium conditions to a system of three differential equations. To this end, recall that $Q_{0}>1$ and $Q_{+}>1$ are satisfied, which implies that locally at both stationary equilibria under consideration it holds that $Q(t)>1$. The equilibrium dynamics are therefore described by

$$
\begin{aligned}
\dot{k}(t) & =\xi Q(t) k(t)+q(t)-\delta k(t), \\
\dot{c}(t) & =[r(t)-\rho] c(t) / \theta \\
\dot{Q}(t) & =[r(t)+\delta] Q(t)-\xi Q(t)[Q(t)-1]-\alpha k(t)^{\alpha-1}, \\
\dot{q}(t) & =r(t) q(t)-[Q(t)-1] q(t), \\
c(t) & =k(t)^{\alpha}-\xi Q(t) k(t)-q(t) .
\end{aligned}
$$


Equation (24) follows from (12) and (14), Eq. (25) is just a repetition of the Euler equation (2), Eq. (26) follows from (22), Eq. (27) follows from (10), and Eq. (28) combines (14), (16), and (21). Conditions (24)-(28) are the five equations referred to by Miao and Wang (2018) in their Appendix D; see Footnote 1 of the present paper.

Lemma 2 In every equilibrium, in which $Q(t)>1$ holds for all $t$, the interest rate satisfies $r(t)=R(k(t), Q(t), q(t))$, where the function $R$ is given by

$$
\begin{aligned}
R(k, Q, q) & \frac{\{\rho-\alpha \theta[\delta-\xi(1+Q)]\} k^{\alpha}+\alpha \theta k^{\alpha-1} q-\xi(\rho+\theta \xi) Q k-[\rho+\theta-\theta(1-\xi) Q] q}{k^{\alpha}-\xi(1-\theta) Q k-(1-\theta) q} .
\end{aligned}
$$

Proof Differentiating Eq. (28) with respect to time $t$ and using Eqs. (24)-(27) to eliminate the time derivatives $\dot{k}(t), \dot{c}(t), \dot{Q}(t)$, and $\dot{q}(t)$ we obtain an equation that is linear in the interest rate $r(t)$. Solving this equation we obtain $r(t)=\tilde{R}(k(t), c(t), Q(t), q(t))$ with $\tilde{R}$ defined by

$$
\begin{aligned}
& \tilde{R}(k, c, Q, q) \\
& \quad=\frac{\alpha \theta[\xi(1+Q)-\delta] k^{\alpha}+\alpha \theta q k^{\alpha-1}-\theta \xi^{2} Q k+\rho c-\theta[1-(1-\xi) Q] q}{c+\theta(\xi Q k+q)} .
\end{aligned}
$$

Using (28) once more to eliminate $c(t)$ from the above expression it follows that $r(t)=R(k(t), Q(t), q(t))$ with $R$ defined in (29).

Using the above lemma, we can write the equilibrium dynamics as

$$
\left(\begin{array}{c}
\dot{k}(t) \\
\dot{Q}(t) \\
\dot{q}(t)
\end{array}\right)=F(k(t), Q(t), q(t)),
$$

where $F: \mathbb{R}_{+}^{3} \mapsto \mathbb{R}^{3}$ is defined by its three components

$$
\begin{aligned}
& F^{1}(k, Q, q)=\xi Q k+q-\delta k \\
& F^{2}(k, Q, q)=[R(k, Q, q)+\delta] Q-\xi Q(Q-1)-\alpha k^{\alpha-1} \\
& F^{3}(k, Q, q)=R(k, Q, q) q-(Q-1) q
\end{aligned}
$$

The Jacobian matrix of this system in a fixed point $(k, Q, q)$ is

$$
\begin{aligned}
& J(k, Q, q) \\
& \quad=\left(\begin{array}{ccc}
\xi Q-\delta & \xi k & 1 \\
R_{1} Q+\alpha(1-\alpha) k^{\alpha-2} & R_{2} Q+\rho+\delta-\xi(2 Q-1) & R_{3} Q \\
R_{1} q & R_{2} q-q & R_{3} q+1+\rho-Q
\end{array}\right),
\end{aligned}
$$

where we have used the fact that $R(k, Q, q)=\rho$ holds for every stationary equilibrium and where we have omitted the arguments of the partial derivatives of $R$ for simplicity. 
In the following two theorems we characterize the eigenvalues of the Jacobian matrix $J(k, Q, q)$ at the two stationary equilibria.

Theorem 2 Suppose that $\xi<\delta$ is satisfied. The eigenvalues of the Jacobian matrix $J\left(k_{0}, Q_{0}, q_{0}\right)$ are

$$
1+\rho-\delta / \xi, \lambda_{1}, \lambda_{2}
$$

where $\lambda_{1}$ and $\lambda_{2}$ are the roots of the quadratic polynomial

$$
\begin{aligned}
\mathcal{P}_{0}(\lambda)= & \lambda^{2}-\lambda\left[\delta R_{2}\left(k_{0}, Q_{0}, q_{0}\right) / \xi+\rho+\xi-\delta\right] \\
& -\delta R_{1}\left(k_{0}, Q_{0}, q_{0}\right) k_{0}-\alpha(1-\alpha) \xi k_{0}^{\alpha-1}
\end{aligned}
$$

The eigenvalues $\lambda_{1}$ and $\lambda_{2}$ are real and of opposite signs.

Proof Since $q_{0}=0$ and $Q_{0}=\delta / \xi$ we have

$$
J\left(k_{0}, Q_{0}, q_{0}\right)=\left(\begin{array}{ccc}
0 & \xi k_{0} & 1 \\
\delta R_{1} / \xi+\alpha(1-\alpha) k_{0}^{\alpha-2} & \delta R_{2} / \xi+\rho+\xi-\delta & \delta R_{3} / \xi \\
0 & 0 & 1+\rho-\delta / \xi
\end{array}\right) .
$$

This proves immediately that one of the eigenvalues is $1+\rho-\delta / \xi$ and that the remaining two eigenvalues are the roots of $\mathcal{P}_{0}(\lambda)$, which is the characteristic polynomial of the upper-left $2 \times 2$ submatrix of $J\left(k_{0}, Q_{0}, q_{0}\right)$. To prove the last statement of the theorem it suffices to show that

$$
\mathcal{P}_{0}(0)=-\delta R_{1}\left(k_{0}, Q_{0}, q_{0}\right) k_{0}-\alpha(1-\alpha) \xi k_{0}^{\alpha-1}<0
$$

holds. Using Theorem 1(b) and Lemma 2 it is straightforward to verify that

$$
\mathcal{P}_{0}(0)=-\frac{\delta^{2}(1-\alpha)(\rho+\xi)\{\rho+\xi[1-\alpha(1-\theta)]\} k_{0} c_{0}}{\alpha \xi\left(c_{0}+\delta \theta k_{0}\right)^{2}},
$$

which is obviously negative for all feasible parameter values.

Theorem 3 Suppose that $\xi<\delta /(1+\rho)$ is satisfied. The Jacobian matrix $J\left(k_{+}, Q_{+}, q_{+}\right)$ has exactly one stable eigenvalue.

Proof Using $Q_{+}=1+\rho$ we obtain

$$
\begin{aligned}
J & \left(k_{+}, Q_{+}, q_{+}\right) \\
\quad & \left(\begin{array}{ccc}
\xi(1+\rho)-\delta & \xi k_{+} & 1 \\
R_{1}(1+\rho)+\alpha(1-\alpha) k_{+}^{\alpha-2} & R_{3}(1+\rho)+\rho+\delta-\xi(1+2 \rho) & R_{4}(1+\rho) \\
R_{1} q_{+} & R_{3} q_{+}-q_{+} & R_{4} q_{+}
\end{array}\right) .
\end{aligned}
$$

The characteristic polynomial of this matrix has the form

$$
\mathcal{P}_{+}(z)=-z^{3}+D_{2} z^{2}+D_{1} z+D_{0}
$$


where

$$
\begin{aligned}
D_{2}= & \frac{1}{\Delta}\left\{\alpha \delta^{2}(1+\rho) \theta+(1+\rho)(1-\xi)\left[\rho^{2}(1-\xi)-\alpha \theta \xi(1+2 \rho)\right]\right. \\
& \left.+\delta\left[\alpha \theta(1-2 \xi)+\rho^{2}(1+\alpha \theta)(1-\xi)+\rho(1-\alpha-\xi+\alpha \xi+\alpha \theta(3-4 \xi))\right]\right\} \\
D_{1}= & \frac{1}{\Delta}\left\{\delta^{3}[1+\rho-\alpha(1+\rho \theta)]\right. \\
& +\rho(1+\rho)^{2}(1-\xi) \xi[\alpha(1-\xi)(\theta-\rho+2 \rho \theta)+(1+\rho) \xi] \\
& +\delta^{2}\left[\rho^{2}(2-3 \xi-\alpha \xi-3 \alpha \theta+4 \alpha \theta \xi)-\left(1-\alpha^{2}\right) \xi\right. \\
& \left.+\rho\left(2-4 \xi+\alpha^{2} \xi-\alpha-2 \alpha \theta+3 \alpha \theta \xi\right)\right] \\
& +\delta(1+\rho)\left[(1-\alpha) \xi^{2}-\rho\left(\xi+2 \alpha \xi-\alpha \xi^{2}-\alpha^{2} \xi+\alpha^{2} \xi^{2}-3 \xi^{2}\right.\right. \\
& \left.\left.+\alpha \theta-4 \alpha \theta \xi+3 \alpha \theta \xi^{2}\right)\right] \\
& \left.+\delta(1+\rho) \rho^{2}\left[1-3 \xi+3 \xi^{2}-\alpha(1-\xi)(2 \xi+2 \theta-5 \theta \xi)\right]\right\} \\
& (1-\alpha)(1+\rho)[\delta-\xi(1+\rho)][\delta+\rho(1-\xi)] \\
D_{0}= & \frac{\Delta}{\Delta}\{(1-\alpha) \delta \\
& +\rho[1+\rho+\delta-\xi(1+\rho)]\}
\end{aligned}
$$

and

$$
\Delta=\delta[1-\alpha(1-\theta)]+\rho[1+\rho+\delta-\xi(1+\rho)] .
$$

The assumption $\xi<\delta /(1+\rho)$ implies that

$$
\begin{aligned}
& \Delta>0, \\
& \delta+\rho(1-\xi)>0, \\
& (1-\alpha) \delta+\rho[1+\rho+\delta-\xi(1+\rho)]>0 .
\end{aligned}
$$

Hence, it follows that $D_{0}<0$, which proves that $\mathcal{P}_{+}$has at least one negative real root. It also follows that there cannot be two stable roots (counting multiplicities). To complete the proof of the theorem it is therefore sufficient to rule out that there are three stable roots.

From the results in Strelitz (1977) and Weisstein (undated) it follows that $\mathcal{P}_{+}$has three stable roots if and only if $D_{0}<0, D_{1}<0, D_{2}<0$, and $D_{0}+D_{1} D_{2}>0$. It is therefore sufficient to prove that $D_{2}>0$ holds which, because of $\Delta>0$, is equivalent to $D_{2} \Delta>0$. It will be convenient to write $D_{2} \Delta=h(\alpha, \theta, \xi) .{ }^{8}$ First of all note that $h(\alpha, \theta, \xi)$ is linear with respect to $\alpha$ and that

\footnotetext{
8 Dependence of $D_{2} \Delta$ on the remaining parameters $\delta$ and $\rho$ is not important for the following argument, which is why we do not include these parameters as arguments of the function $h$.
} 


$$
h(0, \theta, \xi)=\rho(1+\rho)(1-\xi)[\delta+\rho(1-\xi)] \geq 0
$$

holds. Since $\alpha \in(0,1)$ is satisfied, it is sufficient to prove that $h(1, \theta, \xi)$ is nonnegative. It holds that

$$
\begin{aligned}
h(1, \theta, \xi)= & \rho^{3}+\rho^{2}(1+\delta+\delta \theta)+\rho \delta \theta(3+\delta)+\delta \theta(1+\delta) \\
& -\left[2 \rho^{3}+(1+2 \delta) \theta+(3+4 \delta) \rho \theta+(2+\delta) \rho^{2}(1+\theta)\right] \xi \\
& +(1+\rho)\left(\rho^{2}+2 \theta \rho+\theta\right) \xi^{2},
\end{aligned}
$$

and

$$
h(1,0, \xi)=\rho^{2}(1-\xi)[\delta+(1-\xi)(1+\rho)] .
$$

We observe that $h(1, \theta, \xi)$ is linear with respect to $\theta$ and that the assumption $\xi(1+$ $\rho)<\delta$ implies that $h(1,0, \xi)>0$ holds. Consequently, it is sufficient to prove that $h_{2}(1, \theta, \xi)$ is positive. We have

$h_{2}(1, \theta, \xi)=\delta^{2}(1+\rho)+\delta\left[1+3 \rho-\left(\rho^{2}+4 \rho+2\right) \xi\right]-\left(1+3 \rho+2 \rho^{2}\right) \xi(1-\xi)$.

The function $h_{2}(1, \theta, \xi)$ is independent of $\theta$ (because of linearity of $h(1, \theta, \xi)$ with respect to $\theta$ ) and it is a strictly convex quadratic function of $\xi$. This quadratic function attains its minimum at

$$
\bar{\xi}=\frac{1+3 \rho+2 \rho^{2}+\delta\left(2+4 \rho+\rho^{2}\right)}{2\left(1+3 \rho+2 \rho^{2}\right)}
$$

and it holds that $\bar{\xi}>\delta /(1+\rho)$. This implies that $h_{2}(1, \theta, \xi)$ is strictly decreasing with respect to $\xi \in(0, \delta /(1+\rho))$. Since $h_{2}(1, \theta, \delta /(1+\rho))=\delta \rho(1+\rho)>0$ it follows that $h_{2}(1, \theta, \xi)>0$ holds for all feasible parameter values. This completes the proof of $D_{2}>0$.

\section{Discussion}

We have shown that the results from Miao and Wang (2018) on the dynamics locally around stationary equilibria in an economy with risk neutral households carry over to the case of risk averse households. More specifically, if the fraction $\xi$ of capital that can be pledged as collateral is sufficiently high, only a bubbleless stationary equilibrium exist and it is saddle point stable. If the parameter $\xi$ falls below a certain threshold value, a bubbly stationary equilibrium bifurcates from the bubbleless one. In the course of this bifurcation the bubbly equilibrium inherits the saddle point stability whereas the bubbleless stationary equilibrium becomes indeterminate. The situation is therefore completely analogous to that in the overlapping generations model of Tirole (1985): the distinction between $\xi$ being above or below the threshold in the present paper corresponds to the distinction between the bubbleless steady state capital stock being below or above the Golden Rule capital stock in Tirole (1985). 
The stability properties derived in Miao and Wang (2018) and the present paper imply that there exist equilibria converging to the stationary ones, no matter whether they contain bubbles or not. Since the stationary equilibrium capital stocks are smaller than the Golden Rule capital stock (see Lemma 1), all those convergent equilibria are dynamically efficient. In the model of Tirole (1985), on the other hand, existence of bubbly equilibria is only possible if the bubbleless equilibrium is dynamically inefficient.

Acknowledgements Open access funding provided by University of Vienna.

Open Access This article is distributed under the terms of the Creative Commons Attribution 4.0 International License (http://creativecommons.org/licenses/by/4.0/), which permits unrestricted use, distribution, and reproduction in any medium, provided you give appropriate credit to the original author(s) and the source, provide a link to the Creative Commons license, and indicate if changes were made.

\section{References}

Miao J, Wang P (2012) Bubbles and total factor productivity. Am Econ Rev 102:82-87

Miao J, Wang P (2014) Sectoral bubbles, misallocation, and endogenous growth. J Math Econ 53:153-163

Miao J, Wang P (2018) Asset bubbles and credit constraints. Am Econ Rev 108:2590-2628

Strelitz S (1977) On the Routh-Hurwitz problem. Am Math Mon 84:542-544

Tirole J (1985) Asset bubbles and overlapping generations. Econometrica 53:1071-1100

Weisstein EW (undated) Stable polynomial. Wolfram MathWorld. http://mathworld.wolfram.com/ StablePolynomial.html. Accessed July 2018

Publisher's Note Springer Nature remains neutral with regard to jurisdictional claims in published maps and institutional affiliations. 